DOE/ER/ $14024--3$

DE92 005301

\title{
DYNAMICS OF CHARGE-TRANSFER EXCITED STATES RELEVANT TO PHOTOCHEMICAL ENERGY CONVERSION
}

\author{
Progress Report
}

for the Period June 1, 1991 to November 15, 1991

Edward C. Lim

The University of Akron

Akron, $\mathrm{OH} 44325$

November 1991

Prepared for

THE U. S. DEPARTMENT OF ENERGY

AGREEMENT NO. DE-FG02-89-ER14024
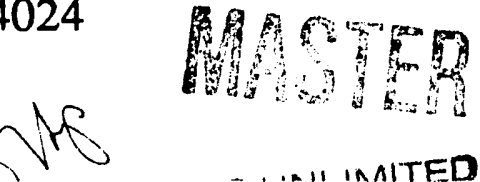

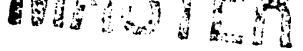




\section{NOTICE}

The report was prepared as an account of work sponsored by the United States Government. Neither the United States nor the Department of Energy, nor any of their employees, nor any of their contractors, subcontractors, or their employees, makes any warranty, express or implied, or assumes any legal liability or responsibility for the accuracy, completeness, or usefulness of any information, apparatus, product or process disclosed or represents that its use would not infringe privately-owned rights. 


\section{Progress Report}

The primary objective of the research program is to gain a fundamental understanding of the factors governing the efficiency of excited-state CT interactions between two chromophores that are brought together in close proximity, either by a very short covalent linkage or by ground-state complex formation. CT and van der Waals ( $\mathrm{dWW}$ ) interactions in covalently bonised bichromophoric compounds in condensed phase, as well as those in $v d W$ complexes in supersonic jets, are being investigated using laser-based techniques under a variety of experimental conditions.

This progress report is divided into three parts, according to the class of molecular systems and the phase (liquid vs. gas) in which the excited-state interactions are probed. The first is concerned with the excited states of bridged diaryl compounds in the condensed phase. The second involves the excited states of vdW complexes in supersonic jets. Finally, the third is concerned with the excited states of electron donor-acceptor (EDA) systems in both the condensed phase and supersonic jets. In each of these studies, we are concerned with the interchromophore interactions ranging from weak $v d W$ forces to strong CT forces, and the factcrs determining whether the interaction forces are weak or strong in related molecules.

\section{I.- Excited States of Bridged Diaryl Compounds in Fluid Solutions}

We have carried out a systematic study of intramolecular photoassociation and photoinduced CT in bichromophoric systems of the general structure $M-X-M$, where two identical aromatic hydrocarbons $(M)$ are joined to each other by a single bridging group $\left(\mathrm{X}=\mathrm{CH}_{2}, \mathrm{O}, \mathrm{NH}\right.$, etc. $)$. These molecular systems are especially well suited for studying intramolecular CT. and other interactions in the excited state for several reasons. Structurally, this is the simplest class of bridged diaryl compounds with limited ranges of accessible conformations. Moreover, the systems provide a homologous series in which a single factor, such as the nature of the linking bridge or the size of the aromatic moiety, can be varied systematically. Relative orientation of the two chromophores can also be easily changed by attaching the bridging group at different positions on the aromatic moiety (e.g., $\alpha$ or $\beta$ position of naphthalene). Equally important, the redox potential and excited-state 
energies are sufficiently different for molecules with different aromatic moieties to provide a wide range of free energy, as well as reorganization energy. Our work with these molecular systems was initially concerned with the question of whether biphotonic excitation of both chromophores in the same molecule, leading to the possible formation of an intramolecular bicimer (short for biexcited dimer), can induce CT between the identical halves via symmetry breaking. Consistent with this supposition, 1,1'-diarylmethanes (phenyl, naphthyl, and anthryl) exhibit, in polar solvents, transient absorption spectra which could be reproduced by superposition of the cation and anion radical spectra of the respective aromatic hydrocarbon. It was, however, not possible to rule out the formation of the radical ions via twophoton ionization of one moiety, followed by an electron attachment to the second. Thus, it was essential to first probe spectroscopic and photophysical properties of these bichromophoric compounds, subsequent to the monophotonic excitation of the molecule to its lowest excited singlet state.

Studies on dinaphthylmethanes $\left(1,1^{\prime}\right.$ and $\left.2,2^{\prime}\right)$, dinaphthylethers $\left(1,1^{\prime}\right.$ and $\left.2,2^{\prime}\right)$ and dinaphthylamines $\left(1,1^{\prime}\right.$ and $\left.2,2^{\prime}\right)$, which have just been completed, demonstrate that the intermoiety interactions can occur in the lowest triplet state through weak $\mathrm{vdW}$ forces, or in the lowest excited singlet state through strong CT forces, depending upon the nature of the linking group, the position of linkage, and the solvent polarity. In dinaphthylmethanes $\left(1^{\prime} 1^{\prime}\right.$ and $\left.2,2^{\prime}\right)$ and dinaphthylethers $\left(1,1^{\prime}\right.$ and $\left.2,2^{\prime}\right)$, monophotonic excitation of a molecule into its lowest excited singlet state leads to the formation of an intramolecular triplet excimer (species stabilized by vdW forces), which can be detected by its unique absorption and phosphorescence, and by a distinctive fluorescence resulting from bimolecular annihilation of the species.

Unlike DNM (dinaphthylmethanes) and DNE (dinaphthylethers), the intermoiety interactions in dinaphthylamines occur in the lowest excited singlet state, with dynamics which greatly depend on the nature of the solvent and the position of the $\mathrm{N}-\mathrm{H}$ linkage ( $\alpha$ vs. $\beta$ ). In nonpolar solvents, $\beta$-DNA $\left(2,2^{\prime}-\right.$ di- $\beta$-naphthylamine) does not exhibit photoassociation in the singlet manifold, and the observed fluorescence is similar to that of the $\beta$-naphthylamine, both in spectral shape and temporal decay profile. As in the case of DNM and DNE, $\beta$-DNA in nonpolar solvents forms triplet excimers, following intersystem crossing of "non-interesting" singlets to the triplet manifold. In strongly 
polar solvents, on the other hand, the molecule forms an intramolecular CT state, as evidenced by the appearance of new transient absorption and the strong solvent-polarity dependence of the Stokes shift of the fluorescence. Since the symmetry breaking, leading to the formation of the CT state, renders the two halves of the DNA non-equivalent, the term "exciplex" is more appropriate than "excimer" in describing the intramolecular dimeric species formed in the excited singlet state. The formation of the intramolecular CT exciplex occurs more efficiently in $\alpha$-DNA (1,1'-di- $\alpha$-naphthylamine) than in $\beta$-DNA. Thus, $\alpha$-DNA exhibits the CT exciplex even in low-polarity solvents, and the time evolution of the fluorescence is too fast to follow, in fluid solution at room temperature, with our instrument response time of about 70 picoseconds. The increase in the dipole moment in going from the ground state to the lowest excited singlet state, deduced from the solvatochromic shift of the fluorescence maximum, is also substantially greater $(\Delta \mu=17 \mathrm{D})$ for $\alpha$ DNA than for $\beta$-DNA $(\Delta \mu \approx 11 D)$.

\section{Excited States of $\mathrm{vdW}$ Complexes in Supersonic Jets}

In condensed phase, the experimentally determined rate constants of bimolecular reactions in general are average over all possible solute/solvent conformations, wide ranges of distances and orientations of reactants, and ranges of internal energies characteristic of the temperature. As a result of this averaging, condensed-phase studies of CT processes tend to mask the importance of the electronic and Franck-Condon factors that control the rate of electron transfer. A detailed understanding of CT dynamics, and solvent effects thereon, requires the study of CT in systems with known geometries and levels of internal energy. Supersonic jet expansion provides opportunities to place reactants in a definite relative arrangement, and excite them into a specific quantum state of the system. Effects of solvation can also be probed in supersonic jets by adding in solvent molecules, to complex with the reactants, one molecule at a time. With these considerations in mind, we have initiated a systematic study of single-vibronic-level (SVL) dependence of exciplex formation in the electronically excited state of jet-cooled $v d W$ complexes.

Writing the wavefunction of the exciplex in the most general form 


$$
\Phi=a \Phi\left(M_{1} * M_{2}\right)+b \Phi\left(M_{1} M_{2}^{*}\right)^{\prime}+c \Phi\left(M^{+} M^{-}\right)+d \Phi\left(M^{-} M^{+}{ }_{2}\right)
$$

one may classify exciplexes into two limiting cases: (1) those formed between two similar molecules; $|a|$ - $|b|$ and $|c|$ - $|d|$, and (2) those formed between a molecule with a low ionization potential and a molecule with a large electron affinity; $|a|,|b|$ * $|c|$, $|d|$. In order to deduce factors that control the efficiency of exciplex formation for these limited cases, we have studied laser-induced fluorescence of the vdW complexes composed of fluorene and substituted benzenes (case 1), and those formed between 1-cyanonaphthalene and various aliphatic amines (case 2).

This study has shown that for the fluorene/substituted benzenes vdW complexes, the exciplex formation following the electronic excitation of fluorene (F) is observed only for substituted benzene (B) whose $S_{1}$ (lowest excited singlet state) electronic origin is similar in energy to that of the lower-lying $S_{1}$ fluorene. The results suggest that the exciplex formation, as monitored by the appearance a broad structureless fluorescence red shifted from the fluorescence of the LE state of the complex $\left(F^{*} B\right)$, is driven largely by exciton resonance $\left(\mathrm{F}^{*} \mathrm{~B} \leftrightarrow \mathrm{FB}^{*}\right)$ between the component molecules. This conjecture is supported by the observation that the efficiency of the exciplex formation is greater for fluorene-h10 than for fluorene-d10 with greater energy mismatch (due to the smaller zero-point energy of fluorene-d 10 relative to fluorene-h10). The appearance of a long progression in the lowfrequency $\left(\sim 5 \mathrm{~cm}^{-1}\right)$ intermoiety vibrations, both in the excitation spectra of the LE fluorescence and exciplex fluorescence, makes it possible to probe the efficiency of the exciplex formation as a function of the number of quanta of the intermolecular vibrations excited. It was observed that the ratio of the intensity of the exciplex fluorescence to that of the LE fluorescence increases with increasing quanta of the intermolecular vibrations initially deposited in the LE state of the fluorene/tetrachlorobenzene complex. This observation, which is the first of its kind, can be rationalized within the framework of Fermi's golden rule applied to electronic relaxation processes, if we treat the exciplex formation as a radiationless transition from a single vibronic level of the LE state to a dense manifold of the isoenergetic vibrational levels of the exciplex state. For such a transition, theory predicts that the rate of the LE $\rightarrow$ exciplex transformation increases with increasing vibrational excitation of 
good promoting modes, as well as good accepting modes. This line of argument would imply that the progressicn-forming intermolecular vibration observed in the fluorescence excitation spectra is the mode that is closely related to the reaction coordinates for the exciplex formation.

For the case of the $\mathrm{vdW}$ complexes of 1-cyanonaphthalene (CNN) with aliphatic amines, belonging to case 2 , the efficiency of the exciplex formation was found to depend both on the ionization potential (IP) of the amine (electron donor) and on the excess vibrational energy of the electronically excited CNN (electron acceptor) moiety. Thus, the complex involving the donor molecule with the highest IP (viz., ammonia) does not exhibit exciplex formation even at high excess energies, while that involving the donor molecule with the lowest IP (via., tributylamine) forms an exciplex even at zero excess energy. For donors with intermediate IPs (dimethylamine, diethylamire, trimethylamine, and tripropylamine), the exciplex formation from the LE state $\left(A^{*} D\right)$ of the $V d W$ complex requires excess energies above a certain threshold value, which decreases with increasing donor strength. These results indicate that the exciplex formation in these systems is driven primarily by $C T$ forces, and that the energy of the $C T$ state $A^{-} D^{+}$relative to that of the initially excited LE state determines the efficiency of the exciplex formation (via their effects on the reaction barrier for the $A^{*} D \rightarrow A^{-} D^{+}$ transformation).

\section{I. Excited States of EDA Systems}

A. Exciplexes in Solution

Electronically excited EDA systems are generally classified as electronically excited CT (or EDA) complexes if they exhibit a stable ground state with a characteristic CT absorption band, or as exciplexes if they do not. Since the ground state of an exciplex is repulsive (i.e., not stable), the formation of the species requires the association of an electronically excited acceptor (or donor) with the ground-state donor (or acceptor) during the lifetime of the electronically excited moiety. Depending upon the nature of the donor and acceptor molecules, the excited state of the EDA system can behave as an exciplex, an electronically excited CT complex or both.

It is generally assumed that the formation and decay of the exciplexes in condensed phase involves three steps. In the first step, $A^{*}$ and $D$ (or $A$ and $D^{*}$ ) 
diffuse together to form an encounter complex $\left(A^{*} D\right)$. In the second step, the encounter complex undergoes a reorganization towards a transition state in which $C T$ takes place to form an exciplex, $\left(A^{-} D^{+}\right)$. Finally, the exciplex decays by dissociation into solvated product ions $A^{-}$and $D^{+}$. or by electronic relaxations. Although the assumption regarding the formation of the encounter complex is perfectly reasonable, and routinely used in various kinetic schemes involving exciplex-mediated CT processes, no spectroscopic evidence exists concerning the existence of this species. Identification and characterization of the encounter complex, if possible, will lead to detailed insights into $\mathrm{CT}$ processes occurring through exciplex mediation. Conceptually, the encounter complex is analogous (except for solvation) to the locally excited (LE) state of $A^{*}$ - B of a jet-cooled $v d W$ complex, which correlates with $A^{*}+B$ at large intermolecular separations $\left(R_{A B} \rightarrow \infty\right)$. The fluorescence from the LE state of such a complex is usually red shifted relative to the emission from the uncomplexed $A^{*}$, due to the greater stabilization of the excited state of the complex relative to its ground state. Thus, one signature of the presence of encounter complexes in condensed phase would be the observation of the $A^{*}$ - or $D^{*}$-like fluorescence, which is red shifted with respect to the normal $A^{*}$ or $D^{*}$ fluorescence, following electronic excitation of uncomplexed $A$ or $D$. On the basis of this reasoning, we have very recently carried out time-resolved fluorescence studies on EDA systems composed of 1,2,4,5-tetracyanobenzene (TCNB) as the electron acceptor and benzene and its methylated derivatives as the electron donors. These acceptor/donor pairs have long been known to behave as $\mathrm{CT}$ complexes as well as exciplexes (i.e., the CT excited states of these species can be created either by direct excitation in the CT absorption bands of the ground-state EDA complex or by local excitation of TCNB followed by exciplex formation). Our measurements of the time-resolved fluorescence spectra of the TCNB/toluene system in nonpolar solvents, following picosecond excitation of the uncomplexed TCNB reveal that the initial fluorescence of the uncomplexed TCNB evolves, at longer delay times, into a red-shifted emission bearing a remarkable spectral resemblance to the $\mathrm{T} C \mathrm{NB}$ emicsion. We tentatively assign the red-shifted emission to the fluorescence from the encounter complex $\left(A^{*} D\right)$ formed by the association of $A^{*}$ and $D$. The emission we attribute to $\left(A^{*} D\right)$ is observed only from weak donors (benzene and toluene) in non polar solvents. The dependence of the emission intensity on the donor strength and solvent 
polarity is consistent with, the expectation that the concentration of the encounter complex would be very small when the encounter complex-toexciplex transformation becomes very efficient due to the lowering of the energy of the CT state relative to that of the LE state of TCNB.

The discussion, presented above, also leads to the question of the distinction between an electronically excited EDA complex and the corresponding exciplex (a question that has often been acidiessed in the literature). Perhaps the most dramatic spectroscopic demonstration of their difference came from our work on the EDA system of TCNB ( $\pi$-acceptor) with various ethers (n-donors), where the fluorescence from the CT excited state of the EDA complex has been shown to have very a different energy and lifetime, when compared to the exciplex fluorescence from the same EDA system. For the $b \pi-a \pi$ (viz., aromatic-aromatic) EDA system, the fluorescence from ti.e excited state of the EDA complex is spectrally very similar to the corresponding exciplex fluorescence. Even for these cases, however, the energy-gap dependence of the charge recombination rate of the ion-pair has been shown to be different, depending whether the ion-pair is produced by way of the excitation of the EDA complex or by way of the exciplex route. This difference suggests significant differences in the structure, and solvation, of the two electronically excited species. It has been suggested that the direct excitation in the CT absorption band of the EDA complex leads to contact ion pairs $A^{-} D^{+}$ (i.e., without the intervening solvent molecules between $A^{-}$and $D^{+}$), while the interaction of $A^{*}$ and $D$ leads to solvent-separated ion pairs $A^{-} / D^{+}$. Solvent penetration to the contact ion pairs is thought to lead to the formation of the solvent-separated ion pairs. With the advent of the ultrafast fluorescence upconversion technique capable of resolving subpicosecond emission dynamics, it is now possible to probe, in detail, the mechanism of exciplex formation as well as to probe the difference between an electronically excited $b \pi-a \pi$ EDA complex and the corresponding exciplex. Work along these lines is presently being readied.

\section{B. Electronically-Excited EDA Complexes in Supersonic Jets}

Unlike the excited-state dynamics of jet-cooled vdW complexes about which a number of reports now exist, information available on the spectroscopy and photophysics of EDA complexes in supersonic jets is 
extremely scarce. In fact, the only reported example of the fluorescence from jet-cooled EDA complexes is that between p-xylene (electron donor) and tetracyanoethylene (electron acceptor) which exhibits broad, structureless fluorescence and equally broad fluorescence excitation spectra. Broad, structureless fluorescence excitation spectra were also obtained for the jetcooled EDA complexes of tetracyanobenzene (electron acceptor) with a variety of alkylbenzenes (electron donors), which we investigated several years ago. The result poses an important question: Is the spectral diffuseness homogeneous or inhomogeneous in origin? That is, are the spectral width and diffuseness intrinsic properties of the EDA complex with definite stoichiometry (1:1) and definite structure, as proposed in an earlier work, or are they due to the presence of a large number of EDA complexes with different stoichiometry and conformation? The diffuseness of the excitation spectrum was attributed to a large displacement of the potential energy surface of the excited state relative to that of the ground state, which render the Franck-Condon-allowed absorption to sample a large number of closely spaced vibrational levels high up in the potential well of the excited state. This explanation, while plausible, does not account for the lack of resolved structure even in the longest wavelength tail of the excitation spectrum (where the absorption and fluorescence overlap). An alternative explanation for the diffuse spectrum is also plausible. With the experimental method used to prepare jet-cooled EDA complexes (bubbling the carrier gas through a reservoir of liquid donor), in the two studies, it is not possible to exclude the formation of clusters with many different stoichiometries, energies, and structures. More stringent methods in which a controlled amount of the donor and acceptor molecules are pre-mixed prior to supersonic expansion, and the stoichiometry of the complex is determined by mass analyses, are needed to probe the origin of the spectral diffuseness.

Another issue concerned with the fluorescence excitation spectra of the EDA complexes of TCNB with alkyl benzene donors (toluene and p-xylene) in supersonic jet is the appearance of the two overlapping bands, corresponding to the two CT absorption bands of the complexes. It is not known whether the two CT bands of the complex are due to the presence of two structural isomers, or to two different electronic absorptions of the same species. Because of the proximity of the two highest energy occupied molecular orbitals (with different nodal properties) in alkyl benzenes, it is possible that two CT 
transitions of similar energy, may be observed from one structural isomer of the EDA complexes. In the related TCNE/p-xylene complex, which also exhibits two overlapping CT absorption bands, the presence of two structural isomers has been invoked to account for the two overlapping bands. Both in the TCNB/alkylbenzene and TCNE/p-xylene EDA complexes, the dispersed fluorescence is a mirror image of the lower-energy CT absorption, independent of which CT absorption band is excited. The result is consistent with rapid structural isomerization of the species with the higher-energy absorption band to the species with the lower-energy absorption band, as well as (vide infra) rapid internal conversion from the higher electronic state to the lower electronic state of the same species. Hole-burning spectroscopy is therefore necessary to establish whether the two CT bands are homogeneous or inhomogeneous in origin. Measurements of the pump-probe hole-burning spectra, as well as mass-selected, resonance-enhanced multiphoton ionization spectra are under way to resolve these fundamental issues associated with the electronic absorption spectra of these proto-type $b \pi-a \pi$ EDA complexes in supersonic jet. 


\section{List of Publications Acknowledging the DOE Support}

1. R. J. Locke and E. C. Lim, "Orientational Effect on Intramolecular Triplet Excimer Formation in 1,n-Dinaphthylalkanes", J. Phys. Chem., 93, 6017 (1989).

2. R. J. Locke, "Triplet Excimers: Further Verification by Emission Measurements", Chem. Phys. Lett., 160, 96 (1989).

3. J. Cai and E. C. Lim, "Photochemical Probe of the Conformation of Naphthalene Triplet Excimer: Light-induced Changes in the Phosphorescence of Agosta Pinacol Dimer in Rigid Hydrocarbon Glasses", J. Phys. Chem., 94, 8387 (1990).

4. H. Saigusd and E. C. Lim, "Excited-State Dynamics of Fluorene/Substituted-Benzene van der Waals Complexes in Supersonic Jets: Dependence of Exciplex Formation on the Relative Energies of the Lowest Excited Singlet State of Component Molecules", J. Phys. Chem., 94, 2631 (1990).

5. H. Saigusa and E. C. Lim, "Charge-Tranfer Interactions in 1Cyanonaphthalene van der Waals Complexes with Aliphatic Amines: Dependence of Excited-State Dynamics on Donor Ionization Potential", J. Phys. Chem., 95, 7580 (1991).

6. S. Modiano, J. Dresner and E. C. Lim, "Intramolecular Photoassociation and Photoinduced Charge Transfer in Bridged Diaryl Compounds. I. Photoassociation in the Lowest Triplet State of 2,2'-Dinaphthylether", J. Phys. Chem., 95, 9144 (1991).

7. S. H. Modiano, J. Dresner and E. C. Lim, "Conformational Similarity between Triplet Excimer and Ground-State van der Waals Dimer of Aromatic Hydrocarbons", Chem. Phys. Lett., in press. 
8. S. H. Modiano, J. Dresner and E. C. Lim, "Intramolecular Photoassociation ahd Photoinduced Charge Transfer in Bridged Diaryl Compounds. II. Charge Transfer Interactions in the Lowest Excited Singlet State of Dinaphthylamines, J. Phys. Chem., submitted. 

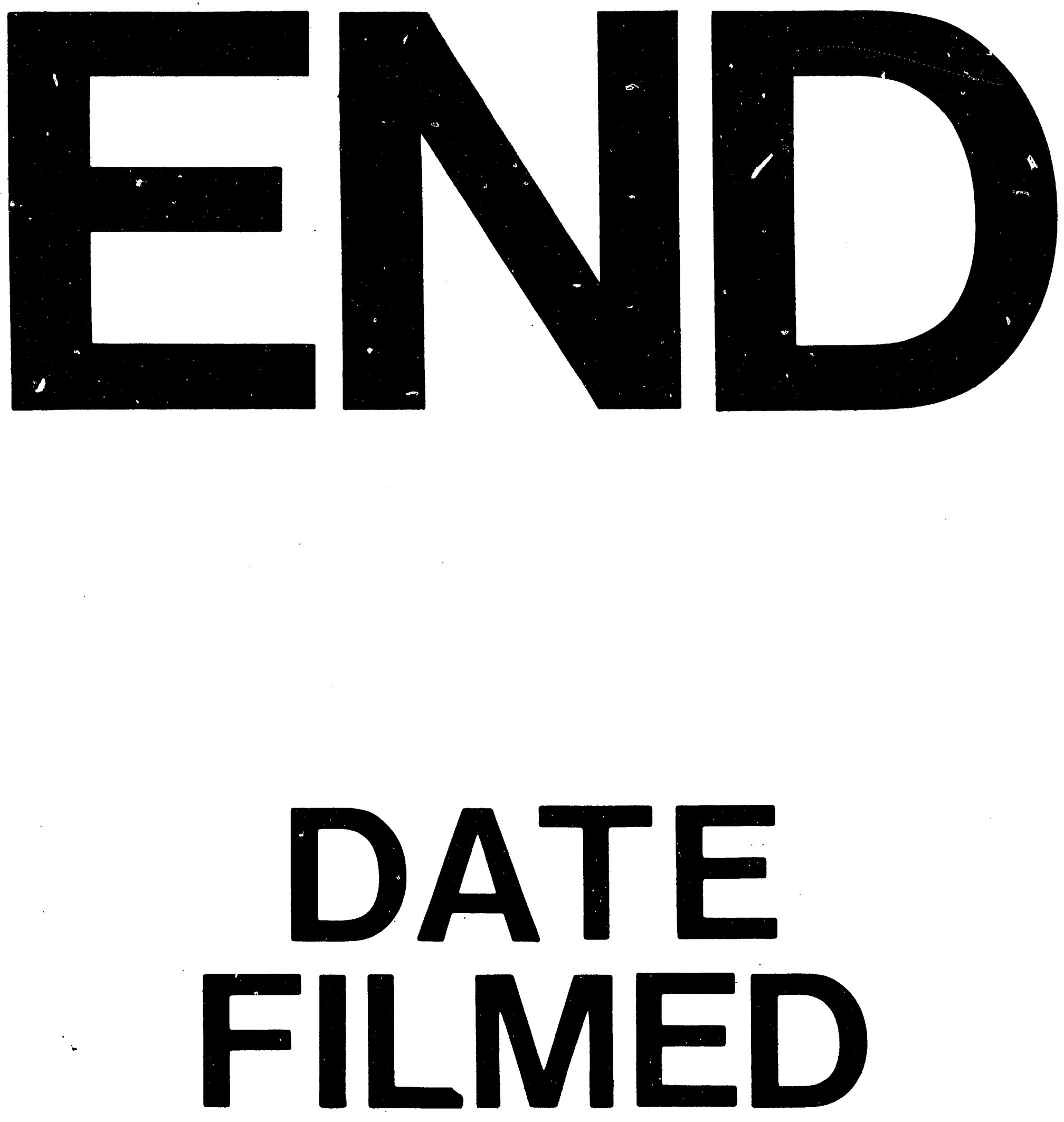

1

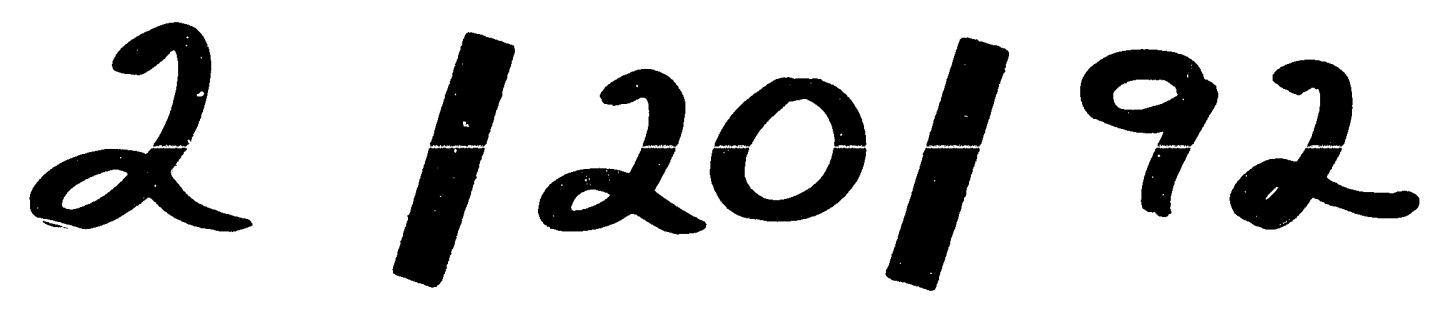


